\title{
ANALYSIS OF A ONE-DIMENSIONAL VARIATIONAL MODEL OF THE EQUILIBRIUM SHAPEL OF A DEFORMABLE CRYSTAL*
}

\author{
Eric Bonnetier ${ }^{1}$, Richard S. FAlK ${ }^{2}$ and Michael A. Grinfeld ${ }^{3}$
}

\begin{abstract}
The equilibrium configurations of a one-dimensional variational model that combines terms expressing the bulk energy of a deformable crystal and its surface energy are studied. After elimination of the displacement, the problem reduces to the minimization of a nonconvex and nonlocal functional of a single function, the thickness. Depending on a parameter which strengthens one of the terms comprising the energy at the expense of the other, it is shown that this functional may have a stable absolute minimum or only a minimizing sequence in which the term corresponding to the bulk energy is forced to zero by the production of a crack in the material.
\end{abstract}

AMS Subject Classification. 49S, 73V25.

Received: August 10, 1998.

\section{INTRODUCTION}

The morphological instabilities of interfaces is a topic of primary interest in physics (e.g., see [4]). Currently, many branches of the natural sciences, including low temperature physics, fracture, crystal growth, epitaxy of nano-scale films, metallurgy, geology, and materials science show a rapidly growing interest in the so called stress driven rearrangement instabilities (SDRI) of surfaces and interfaces in solids. Several examples of the SDRI have been predicted on the basis of Gibbs thermodynamics [5] of heterogeneous systems by studying the positive definiteness of the second energy variations [7] of relevant functionals. At present, some of the predicted instabilities have been confirmed experimentally and found applications in the above mentioned areas.

The thermodynamics of deformable solids with rearrangement leads to certain multi-dimensional variational problems with unknown boundaries and with different specific constraints. Despite its quite simple formulation, the problem in all its entirety is quite complex, and the study of its different features with the help of simpler examples seems quite desirable. Many mathematical aspects of the general problem of thermodynamics of solids with rearrangement can be studied in the framework of the problem of equilibrium shape of deformable crystals formulated in $[7,8]$. This problem is of a certain physical interest on its own in the theory of nano-scale solid crystals [10]. Probably, it is the simplest mathematical problem possessing all of the crucial features of the most general situation. From a mathematical point of view, the problem of the equilibrium shape of a deformable

Keywords and phrases. Equilibrium shape, non-convex energy functional, variational problem.

* The second author was supported by NSF grant DMS-9704556.

1 CNRS-CMAP, École Polytechnique, 91128 Palaiseau, France. e-mail: eopus@cmapx.polytechnique.fr

2 Department of Mathematics, Rutgers University, Piscataway, NJ 08854, USA. e-mail: falk@math.rutgers.edu

${ }^{3}$ Educational Testing Service, Princeton, NJ, USA. e-mail: mgrinfeld@ets.org 
crystal is the natural synthesis of two classical problems of mathematical physics: (i) the problem of equilibrium shape of a rigid crystal of fixed total volume $[11,12]$ and (ii) the problem of the equilibrium of an elastic solid with fixed geometry. The symbiosis, however, gives some qualitatively new features absent in the ingredients. Some valuable analytical facts for this problem can be established with the help of Nozieres's results [13]. Because of the existing difficulties of the general 3D-problem, it is expedient to analyze first its simpler one-dimensional version which is studied in this and in a forthcoming paper. The one-dimensional problem has been formulated in [9] and it allows us to describe some phenomena in elastic shells and strips with movable defects. Below, we present without derivation some facts relating to this problem. Mathematically it is formulated as the minimization of the functional $\mathcal{E}$ depending on two unknown functions: an elastic displacement $u(x)$ and a strip thickness $h(x)$ of one variable $x$ :

$$
\mathcal{E}=\int_{0}^{L}\left[(G / 2) h(x)\left[u^{\prime}(x)\right]^{2}+\sigma \sqrt{1+\left[h^{\prime}(x)\right]^{2}}\right] \mathrm{d} x
$$

where $G>0$ is the elastic modulus, $\sigma>0$ is the surface energy, $u^{\prime}(x)$ is the elastic deformation, and $\sqrt{1+\left[h^{\prime}(x)\right]^{2}} \mathrm{~d} x$ is the length element of an outer boundary of the strip.

We assume that the elastic displacements $u(x)$ and the thickness $h(x)$ are fixed at the end-points, i.e.,

$$
u(0)=U_{0}, \quad u(L)=U_{L}, \quad h(0)=h_{0}, \quad h(L)=h_{L}
$$

and that the total volume of the strip is also fixed, i.e.,

$$
\int_{0}^{L} h(x) \mathrm{d} x=A
$$

For simplicity, we consider the case when

$$
L=1, \quad U_{0}=0, \quad U_{L}=1, \quad h_{0}=1, \quad h_{L}=1, \quad A=1, \quad \sigma=1, \quad G=2 N .
$$

We are thus led to the minimization problem: Find

$$
\inf _{u \in V, h \in H} \mathcal{E}(u, h)=\inf _{u \in V, h \in H} N \int_{0}^{1} h(x)\left[u^{\prime}(x)\right]^{2} \mathrm{~d} x+\int_{0}^{1} \sqrt{1+\left[h^{\prime}(x)\right]^{2}} \mathrm{~d} x .
$$

The set of admissible displacements is $V=\left\{u \in H^{1}(0,1): u(0)=0, u(1)=1\right\}$, and the admissible thicknesses lie in the set $H$ of piecewise $\mathcal{C}^{1}$ functions on $[0,1]$ satisfying

$$
h(x)>0 \text { in }[0,1], \quad h(0)=h(1)=1, \quad \int_{0}^{1} h(x) \mathrm{d} x=1 .
$$

For a given thickness $h \in H$, one can easily check that

$$
u_{h}^{\prime}(x)=\left(\int_{0}^{1}[h(x)]^{-1} \mathrm{~d} x\right)^{-1} \frac{1}{h(x)}
$$

minimizes $\mathcal{E}(u, h)$ in $V$. Thus the displacement can be eliminated in (1.1) and the original problem reduces to minimizing over $h \in H$ the functional

$$
\mathcal{I}(h)=\frac{N}{\int_{0}^{1}[h(x)]^{-1} \mathrm{~d} x}+\int_{0}^{1} \sqrt{1+\left[h^{\prime}(x)\right]^{2}} \mathrm{~d} x .
$$


It is a standard feature in such problems of the calculus of variations, that $\mathcal{I}$ may not attain its infimum on the space of $\mathcal{C}^{1}$ functions. Generally, minimizing sequences may develop oscillations if $\mathcal{I}$ does not have the right properties of convexity. In the case at hand, the second term of $\mathcal{I}$ is convex since

$$
\frac{\partial^{2}}{\partial f^{2}} \sqrt{1+f^{2}}=\left(1+f^{2}\right)^{-3 / 2}>0
$$

but the first term is concave, so the standard direct method is not applicable. Minimizing sequences may also tend to functions which lie outside the initial set of candidates and which are usually less regular. To ensure well-posedness, the problem must be relaxed: a larger class of admissible designs must be allowed and the functional must be extended accordingly [3].

The uniform thickness $h_{0} \equiv 1$ will be called the trivial solution. The value of its energy is $N+1$. One readily checks that $h_{0}$ satisfies the Euler-Lagrange equation associated to (1.3) (however, this is not a sufficient condition for $h_{0}$ to be the absolute minimum!). Many other examples of variational problems whose minimizers do not satisfy the Euler-Lagrange equation can be found in [1]. Because of the nonlocal nature of the term corresponding to the bulk energy in the functional $\mathcal{I}(h)$, the problem discussed here falls outside of the classical theory.

The main results of the paper are the following. In the next section, we consider the standard linearized stability analysis and show that the second variation of the energy for smooth perturbations about the thickness $h_{0} \equiv 1$ is positive for $N \leq 2 \pi^{2}$. However, this does not guarantee that $h_{0} \equiv 1$ is a minimizer even for $N$ in this range. In Section 3 , we show that there exists an $N_{0}>0(\approx 1.159)$ such that for all $N \leq N_{0}, h_{0} \equiv 1$ is an absolute minimizer of the functional $\mathcal{I}$. In Section 4, we prove that for $N \geq 2, \inf _{h \in H} \mathcal{I}(h) \geq 2+\pi / 4$. Then, in the following section, we explicitly construct a minimizing sequence $h_{\epsilon} \in H$ such that $\mathcal{I}\left(h_{\epsilon}\right) \rightarrow 2+\pi / 4$ as $\epsilon \rightarrow 0$, which proves that $\inf _{h \in H} \mathcal{I}(h)=2+\pi / 4$. For this minimizing sequence, the term corresponding to the bulk elastic energy tends to 0 , and the functional reduces to a measure of the length of the curve defined by $h_{\epsilon}$. The disappearance of the bulk energy term is achieved by the production of a crack in the specimen and the energy cost for this is equal to twice the extra length induced by the crack. This is shown explicitly by the construction of a non-parametric curve $\mathcal{H}_{*}$, the length of which equals $2+\pi / 4$, such that $h_{\epsilon}$ converges to $\mathcal{H}_{*}$ a.e. Finally, Section 6 states a relaxation result: since minimizing sequences for $\mathcal{I}$ satisfy natural bounds in the space $B V$ of functions of bounded variation, we define an extension $\mathcal{J}$ of $\mathcal{I}$ on a compact set of $B V$ functions and show that this extension is lower semi-continuous with respect to $B V$.

\section{Stability FOR THE LINEARIZED PROBLEM}

In this section, we consider the standard linearized stability analysis for the trivial solution $h_{0} \equiv 1$ and establish the following result.

Lemma 2.1. If $N \leq 2 \pi^{2}$, and $k$ is a smooth function satisfying $\int_{0}^{1} k \mathrm{~d} x=0$, then $D^{2} \mathcal{I}\left(h_{0}\right) k \otimes k>0$.

Before proving this result, we note that we shall show in Section 4 that $h_{0} \equiv 1$ is not a minimum for values of $N$ which are much lower than $2 \pi^{2}$. This is not contradictory with the lemma, since the linearized analysis only gives information about smooth perturbations. 
Proof. If an admissible function $h$ is smooth, bounded away from 0 , and if $k$ is a smooth function such that $\int_{0}^{1} k \mathrm{~d} x=0$, then

$$
\begin{aligned}
\mathcal{I}(h+\epsilon k)= & \mathcal{I}(h)+\epsilon \int_{0}^{1} \frac{h^{\prime}(x) k^{\prime}(x)}{\sqrt{1+\left[h^{\prime}(x)\right]^{2}}} \mathrm{~d} x+N \epsilon \int_{0}^{1} \frac{k(x)}{[h(x)]^{2}} \mathrm{~d} x\left(\int_{0}^{1}[h(x)]^{-1} \mathrm{~d} x\right)^{-2} \\
& +N \epsilon^{2}\left(\int_{0}^{1} \frac{k(x)}{[h(x)]^{2}} \mathrm{~d} x\right)^{2}\left(\int_{0}^{1}[h(x)]^{-1} \mathrm{~d} x\right)^{-3}-N \epsilon^{2} \int_{0}^{1} \frac{[k(x)]^{2}}{[h(x)]^{3}} \mathrm{~d} x\left(\int_{0}^{1}[h(x)]^{-1} \mathrm{~d} x\right)^{-2} \\
& +\frac{\epsilon^{2}}{2} \int_{0}^{1} \frac{\left[k^{\prime}(x)\right]^{2}}{\left(1+\left[h^{\prime}(x)\right]^{2}\right)^{3 / 2}} \mathrm{~d} x+O\left(\epsilon^{3}\right) .
\end{aligned}
$$

In particular, for the function $h_{0} \equiv 1$, the above becomes

$$
\mathcal{I}\left(h_{0}+\epsilon k\right)=\mathcal{I}\left(h_{0}\right)+\frac{\epsilon^{2}}{2} \int_{0}^{1}\left(\left[k^{\prime}(x)\right]^{2}-2 N[k(x)]^{2}\right) \mathrm{d} x+O\left(\epsilon^{3}\right) .
$$

Hence $h_{0} \equiv 1$ has a lower energy than a smooth perturbation, provided that

$$
\int_{0}^{1}\left(\left[k^{\prime}(x)\right]^{2}-2 N[k(x)]^{2}\right) \mathrm{d} x \geq 0 \quad \forall k \in H_{0}^{1}(0,1) \text { such that } \int_{0}^{1} k \mathrm{~d} x=0 .
$$

Now the functions $e_{n}(x)=\sin (n \pi x), n \geq 1$, form a basis of $H_{0}^{1}(0,1)$ and satisfy

$$
\begin{gathered}
\int_{0}^{1}\left(e_{n}^{\prime}\right)^{2}(x) \mathrm{d} x=n^{2} \pi^{2} \int_{0}^{1} e_{n}^{2}(x) \mathrm{d} x=n^{2} \pi^{2} / 2, \\
\int_{0}^{1} e_{2 n}(x) \mathrm{d} x=0, \quad \int_{0}^{1} e_{2 n+1}(x) \mathrm{d} x=\frac{1}{2 n+1} \frac{2}{\pi} .
\end{gathered}
$$

Let $k(x)=\sum_{n \geq 1} a_{n} e_{n}(x)$. The condition that the average of $k$ vanishes yields

$$
\begin{aligned}
a_{1}^{2} & =\left(\pi / 2 \int_{0}^{1}\left[k(x)-a_{1} e_{1}(x)\right] \mathrm{d} x\right)^{2}=\pi^{2} / 4\left(\int_{0}^{1} \sum_{p \geq 1} a_{2 p+1} e_{2 p+1}(x) \mathrm{d} x\right)^{2} \\
& =\pi^{2} / 4\left(\sum_{p \geq 1} a_{2 p+1} \frac{1}{2 p+1} \frac{2}{\pi}\right)^{2} \leq \sum_{p \geq 1} a_{2 p+1}^{2} \sum_{p \geq 1} \frac{1}{(2 p+1)^{2}} \\
& =\left(\frac{\pi^{2}}{8}-1\right) \sum_{p \geq 1} a_{2 p+1}^{2} .
\end{aligned}
$$

Condition (2.1) reduces to

$$
\sum_{n \geq 1} a_{n}^{2}\left(n^{2} \pi^{2}-2 N\right) \geq 0
$$


Obviously, this condition is fulfilled if $2 N \leq \pi^{2}$. Using (2.1) directly, we see that $N$ must be smaller than $2 \pi^{2}$, since the second eigenfunction $e_{2}$ has a zero average. However, if $\pi^{2}-2 N<0$, the estimate on $a_{1}$ yields

$$
\begin{aligned}
\sum_{n \geq 1} a_{n}^{2}\left(n^{2} \pi^{2}-2 N\right) \geq & \sum_{p \geq 1} a_{2 p+1}^{2}\left[(2 p+1)^{2} \pi^{2}-2 N+\left(\pi^{2}-2 N\right)\left(\pi^{2} / 8-1\right)\right] \\
& +\sum_{p \geq 1} a_{2 p}^{2}\left(4 p^{2} \pi^{2}-2 N\right) .
\end{aligned}
$$

Since the factor in the first sum of the expression on the right hand side is positive for $N \leq 2 \pi^{2}$, we conclude that $D^{2} \mathcal{I}\left(h_{0}\right) k \otimes k$ is positive for $N$ in this range.

\section{Stability OF THE TRIVIAL SOLUTION}

In this section, the trivial solution $h_{0} \equiv 1$ is shown to be the unique minimum of $\mathcal{I}$, if $N$ is sufficiently small. Specifically, we prove the following.

Theorem 3.1. The trivial solution $h_{0} \equiv 1$ is a stable minimum with respect to perturbations of magnitude $k<1$, provided that $N \leq \psi(k) \equiv\left(\sqrt{1+4 k^{2}}-1\right)\left(1-k+k^{2}\right) / k^{2}$. Also, $h_{0}$ is an absolute minimum if $N \leq N_{0} \equiv \inf _{0<k \leq 1} \psi(k) \approx 1.16$.

Proof. We begin by seeking a lower bound for the elastic energy that is quadratic in terms of the maximal and minimal values of $h$, for any admissible thickness $h \in H$. Since $I$ is translation invariant, we can always assume that

$$
h(x)=1+K(x) \geq 1 \quad \text { on }[0, \alpha], \quad h(x)=1-k(x) \leq 1 \quad \text { on }[\alpha, 1] .
$$

The volume constraint on $h$ becomes

$$
\int_{0}^{\alpha} K(x) \mathrm{d} x-\int_{\alpha}^{1} k(x) \mathrm{d} x=0
$$

Let $1+K_{0}$ and $1-k_{0}$ denote the maximum and minimum of $h, 0 \leq K_{0}, 0 \leq k_{0}<1$. Straightforward computations show that if $\lambda_{0}=\left(1-k_{0}\right)^{-1}$,

$$
\frac{1}{1+K} \leq 1-K+K^{2} \quad \forall 0 \leq K \leq K_{0}, \quad \frac{1}{1-k} \leq 1+k+\lambda_{0} k^{2} \quad \forall 0 \leq k \leq k_{0} .
$$

Using (3.1), it follows that

$$
\begin{aligned}
\int_{0}^{1}[h(x)]^{-1} \mathrm{~d} x & =\int_{0}^{\alpha}[1+K(x)]^{-1} \mathrm{~d} x+\int_{\alpha}^{1}[1-k(x)]^{-1} \mathrm{~d} x \\
& \leq \int_{0}^{\alpha}\left[1-K(x)+K^{2}(x)\right] \mathrm{d} x+\int_{\alpha}^{1}\left[1+k(x)+\lambda_{0} k^{2}(x)\right] \mathrm{d} x \\
& \leq 1+\alpha K_{0}^{2}+(1-\alpha) \lambda_{0} k_{0}^{2} .
\end{aligned}
$$

Thus, the elastic part of the energy can be estimated by

$$
\frac{N}{\int_{0}^{1}[h(x)]^{-1} \mathrm{~d} x} \geq \frac{N}{1+\alpha K_{0}^{2}+(1-\alpha) \lambda_{0} k_{0}^{2}} .
$$


On the other hand, a term such as $\int_{0}^{\alpha} \sqrt{1+\left(h^{\prime}\right)^{2}} \mathrm{~d} x$ is the length of a curve that joins the points $(0,1)$ to $(\alpha, 1)$, and that rises up to the level $1+K_{0}$. Suppose that $h(\gamma \alpha)=1+K_{0}$ for some $0<\gamma<1$. The Jensen inequality applied to the convex function $\sqrt{1+x^{2}}$ yields

$$
\int_{0}^{\gamma \alpha} \sqrt{1+\left(h^{\prime}\right)^{2}} \mathrm{~d} x \geq \gamma \alpha\left(1+\left[\int_{0}^{\gamma \alpha} h^{\prime}(x) \frac{\mathrm{d} x}{\gamma \alpha}\right]^{2}\right)^{1 / 2}=\sqrt{\gamma^{2} \alpha^{2}+K_{0}^{2}}
$$

Similarly, on the piece $[\gamma \alpha, \alpha]$, we have

$$
\int_{\gamma \alpha}^{\alpha} \sqrt{1+\left(h^{\prime}\right)^{2}} \mathrm{~d} x \geq \sqrt{(1-\gamma)^{2} \alpha^{2}+K_{0}^{2}}
$$

Using the convexity of $\sqrt{\alpha^{2}+x^{2}}$,

$$
\begin{aligned}
\sqrt{\gamma^{2} \alpha^{2}+K_{0}^{2}}+\sqrt{(1-\gamma)^{2} \alpha^{2}+K_{0}^{2}} & =\gamma \sqrt{\alpha^{2}+\left(\frac{K_{0}}{\gamma}\right)^{2}}+(1-\gamma) \sqrt{\alpha^{2}+\left(\frac{K_{0}}{(1-\gamma)}\right)^{2}} \\
& \geq \sqrt{\alpha^{2}+4 K_{0}^{2}}
\end{aligned}
$$

Hence we obtain

$$
\int_{0}^{\alpha} \sqrt{1+\left(h^{\prime}\right)^{2}} \mathrm{~d} x \geq \sqrt{\alpha^{2}+4 K_{0}^{2}}
$$

A similar estimate holds on the portion $[\alpha, 1]$, with a lower bound $\sqrt{(1-\alpha)^{2}+4 k_{0}^{2}}$. Again, by convexity, adding these two estimates yields

$$
\int_{0}^{1} \sqrt{1+\left(h^{\prime}\right)^{2}} \mathrm{~d} x \geq \sqrt{1+4\left(K_{0}+k_{0}\right)^{2}}
$$

Adding (3.2) and (3.3), we obtain

$$
\mathcal{I}(h) \geq \frac{N}{1+\alpha K_{0}^{2}+(1-\alpha) \lambda_{0} k_{0}^{2}}+\sqrt{1+4\left(K_{0}+k_{0}\right)^{2}} .
$$

As a function of $\alpha$, the first term on the right hand side is increasing if $K_{0}^{2}<\lambda_{0} k_{0}^{2}$. In this case, the lowest value corresponds to $\alpha=0$ so that

$$
\mathcal{I}(h) \geq \frac{N\left(1-k_{0}\right)}{1-k_{0}+k_{0}^{2}}+\sqrt{1+4 k_{0}^{2}}
$$

Thus, $\mathcal{I}(h) \geq \mathcal{I}\left(h_{0}\right)$, provided $N$ is less than

$$
\psi_{m}\left(k_{0}\right)=\frac{\sqrt{1+4 k_{0}^{2}}-1}{k_{0}^{2}}\left(1-k_{0}+k_{0}^{2}\right)
$$

If, on the other hand, $K_{0}^{2} \geq \lambda_{0} k_{0}^{2}$, then the lowest value of the bound corresponds to $\alpha=1$, and then

$$
\mathcal{I}(h) \geq \frac{N}{1+K_{0}^{2}}+\sqrt{1+4 K_{0}^{2}}
$$


The trivial solution achieves the smallest bound, provided $N$ is smaller than

$$
\psi_{M}\left(K_{0}\right)=\frac{\sqrt{1+4 K_{0}^{2}}-1}{K_{0}^{2}}\left(1+K_{0}^{2}\right) .
$$

The first statement of the theorem then follows from the observation that $\psi_{m}(k)<\psi_{M}(k)$ for $k \in(0,1)$. This together with some straightforward computations which show that $\psi_{M}$ is an increasing function of $k$ and that $\inf _{0<k \leq 1} \psi(k) \approx 1.16$ establish the second statement.

\section{A generalized Minimizer FOR $N \geq 2$}

In this section, we compute the infimum of (1.3) for values of $N \geq 2$ and show that it corresponds to the length of a parametric curve representing a generalized thickness.

Theorem 4.1. If $N \geq 2$, then $\inf _{h \in H} \mathcal{I}(h) \geq 2+\pi / 4$. In addition, if $\mathcal{H}_{*}$ is the parametric curve defined by the functions

$$
\begin{array}{ll}
h_{*}(x)=1-\pi / 8+\sqrt{\left(x+x_{*}\right)\left(1-x-x_{*}\right)} & \text { if } 0 \leq x<1-x_{*}, \\
h_{*}(x)=1-\pi / 8+\sqrt{\left(x+x_{*}-1\right)\left(2-x-x_{*}\right)} & \text { if } 1-x_{*} \leq x \leq 1,
\end{array}
$$

and the segment $x=1-x_{*}, 0<y<1-\pi / 8$, with $x_{*}=\left(4-\sqrt{16-\pi^{2}}\right) / 8$, then the infimum of $\mathcal{I}$ corresponds to the length of $\mathcal{H}_{*}$, where the length of the vertical part of $\mathcal{H}_{*}$ is counted twice.

Proof. To establish this result, we rewrite the minimization problem in the following form.

$$
\inf _{h \in H} \mathcal{I}(h)=\inf _{0<\epsilon \leq 1}\left(\inf _{h \in H_{\epsilon}} \mathcal{I}(h)\right)
$$

where $H_{\epsilon}$ is the set of piecewise $\mathcal{C}^{1}$ functions satisfying the constraints (1.2) and

$$
\min _{x \in[0,1]} h(x)=\epsilon .
$$

For $h \in H_{\epsilon}, 1 / h \leq 1 / \epsilon$, so the first term in $\mathcal{I}$ is bounded from below by $N \epsilon$. Thus, we get

$$
\inf _{h \in H_{\epsilon}} \mathcal{I}(h) \geq N \epsilon+\inf _{h \in H_{\epsilon}} \mathcal{L}(h)
$$

where $\mathcal{L}(h) \equiv \int_{0}^{1} \sqrt{1+\left[h^{\prime}(x)\right]^{2}} \mathrm{~d} x$ is the length of the curve $h$. The second term in the above expression is the minimal length of a curve that takes the value 1 at its end points, reaches the value $\epsilon$ as its minimum, and bounds an area equal to 1.

Let $F_{\epsilon}$ be the set of piecewise $\mathcal{C}^{1}$ curves satisfying

$$
f(x) \geq \epsilon \text { in }[0,1], \quad f(0)=f(1)=\epsilon, \quad \int_{0}^{1} f(x) \mathrm{d} x=1 .
$$

To each element $f$ of $F_{\epsilon}$, we associate an element $h$ of $H_{\epsilon}$ in the following way. If $0<\epsilon<1$, the area constraint forces $f$ to take the value 1 . Let $x_{1}$ be the first point where $f=1$. Set

$$
h(x)=f\left(x+x_{1}\right) \quad \text { for } 0 \leq x \leq 1-x_{1}, \quad h(x)=f\left(x-1+x_{1}\right) \quad \text { for } 1-x_{1}<x \leq 1 .
$$


Since the volume constraint and the length of the curve are translation invariant, the function $h$ lies in $H_{\epsilon}$. In a similar fashion, we can associate to a function $h \in H_{\epsilon}$, a function $f \in F_{\epsilon}$ : if $x_{\epsilon}$ is the first point where $h$ achieves the value $\epsilon$, we set

$$
f(x)=h\left(x+x_{\epsilon}\right) \quad \text { for } 0 \leq x \leq 1-x_{\epsilon}, \quad f(x)=h\left(x-1+x_{\epsilon}\right) \quad \text { for } 1-x_{\epsilon} \leq x \leq 1 .
$$

It follows that the infimum of $\mathcal{L}$ can be computed either on $H_{\epsilon}$ or on $F_{\epsilon}$. The latter is a case of the isoperimetric problem. Its solution is described in the next proposition, the proof of which is given in the Appendix.

Proposition 4.2. If $(1-\pi / 8) \leq \epsilon \leq 1$, the curve of minimal length, with value $\epsilon$ at its end points, lying above the value $\epsilon$, and bounding an area equal to 1 , is the arc of circle of radius $R_{\epsilon}$ given by

$$
1-\epsilon=-\sqrt{4 R_{\epsilon}^{2}-1} / 4+R_{\epsilon}^{2} \arcsin \left(1 /\left[2 R_{\epsilon}\right]\right)
$$

Moreover, the corresponding length is $\inf _{f \in F_{\epsilon}} \mathcal{L}(f)=2 R_{\epsilon} \arcsin \left(1 /\left[2 R_{\epsilon}\right]\right)$.

If $0 \leq \epsilon<1-\pi / 8$, the infimum of $\mathcal{L}(f)$ is attained by the curve consisting of the vertical straight lines $[0, y], \epsilon \leq y \leq 1-\pi / 8$ and $[1, y], \epsilon \leq y \leq 1-\pi / 8$, and the half-circle of radius $1 / 2$ joining the point $(0,1-\pi / 8)$ to the point $(1,1-\pi / 8)$. The minimal length is then

$$
\inf _{f \in F_{\epsilon}} \mathcal{L}(f)=2(1-\epsilon)+\pi / 4
$$

Returning to (4.2), we can bound the energy from below by

$$
\begin{array}{ll}
I_{1}(\epsilon)=N \epsilon+2 R_{\epsilon} \arcsin \left(1 /\left[2 R_{\epsilon}\right]\right) & \text { if } 1-\pi / 8 \leq \epsilon \leq 1, \\
I_{2}(\epsilon)=(N-2) \epsilon+2+\pi / 4 & \text { if } 0 \leq \epsilon<1-\pi / 8,
\end{array}
$$

and it follows from (4.1) that

$$
\inf _{h \in H} \mathcal{I}(h) \geq \min \left(\inf _{1-\pi / 8 \leq \epsilon \leq 1} I_{1}(\epsilon), \inf _{0<\epsilon<1-\pi / 8} I_{2}(\epsilon)\right)
$$

We next show that for $N \geq 2$, the infimum in (4.4) is attained at $\epsilon=0$. Differentiating $I_{1}$ with respect to $\epsilon$, we get

$$
\frac{\partial I_{1}}{\partial \epsilon}=N+2\left(\arcsin \left(1 /\left[2 R_{\epsilon}\right]\right)-\frac{1}{\sqrt{4 R_{\epsilon}^{2}-1}}\right) \frac{\partial R_{\epsilon}}{\partial \epsilon}
$$

On the other hand, the definition (4.3) of $R_{\epsilon}$ yields

$$
1=2\left(\frac{R_{\epsilon}}{\sqrt{4 R_{\epsilon}^{2}-1}}-R_{\epsilon} \arcsin \left(1 /\left[2 R_{\epsilon}\right]\right)\right) \frac{\partial R_{\epsilon}}{\partial \epsilon} .
$$

Eliminating $\partial R_{\epsilon} / \partial \epsilon$ between these two relations shows that

$$
\frac{\partial I_{1}}{\partial \epsilon}=N-\frac{1}{R_{\epsilon}} \geq N-2
$$

since $R_{\epsilon} \geq 1 / 2$. Thus, for $N \geq 2, I_{1}$ is an increasing function of $\epsilon$. On the other hand, $I_{2}$ is also increasing in this case, which establishes the result. 

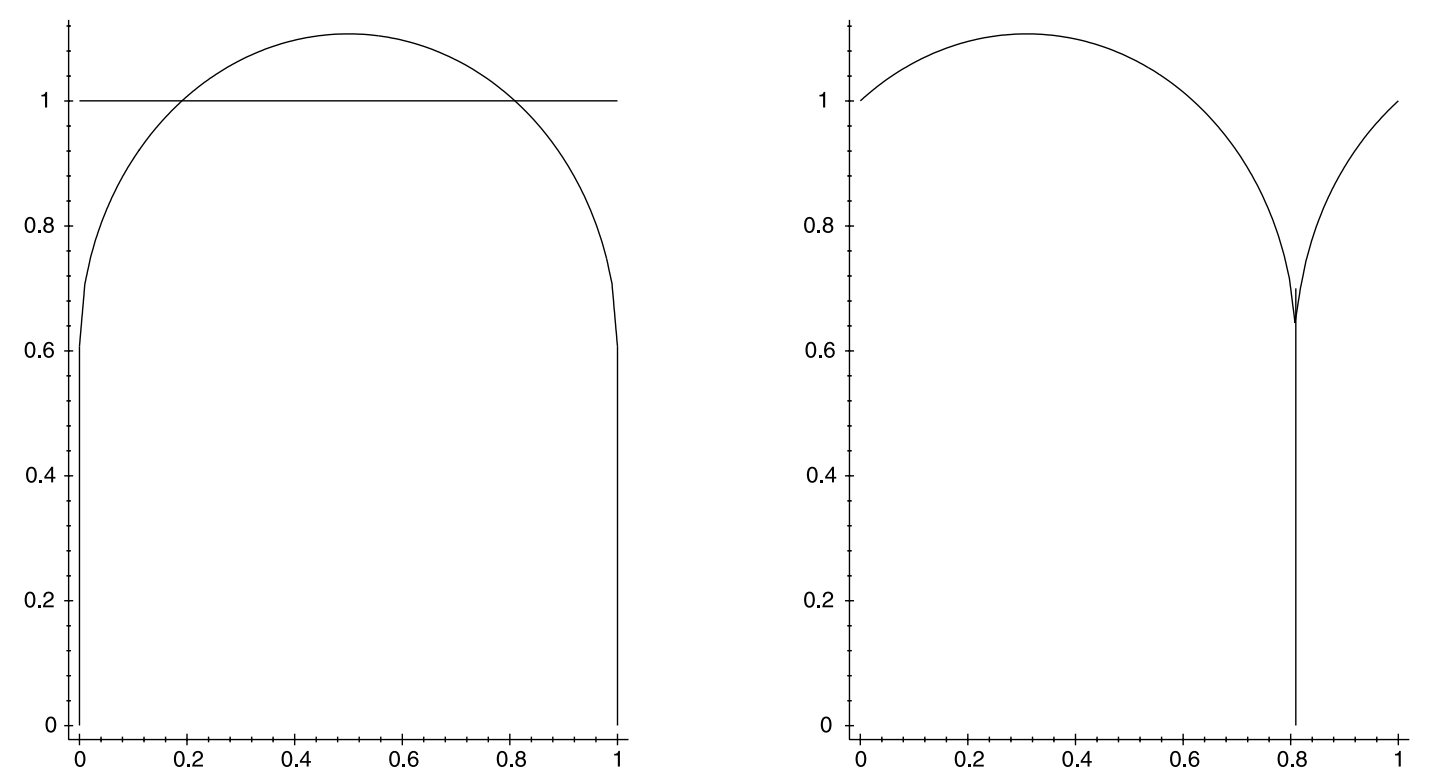

Figure 1. Plot of $\mathcal{F}_{*}(x)$ along with $y=1$ and corresponding plot of $\mathcal{H}_{*}(x)$.

It is then easily checked that $I_{2}(0)=2+\pi / 4$ is the length of the curve $\mathcal{F}_{*}$ defined by the function

$$
f_{*}(x)=1-\pi / 8+\sqrt{x(1-x)} \quad \text { if } 0<x<1
$$

and by the two vertical lines

$$
x=0, \quad 0 \leq y \leq 1-\pi / 8, \quad x=1, \quad 0 \leq y \leq 1-\pi / 8 .
$$

To go back to the original boundary conditions, let $x_{*}=\left(4-\sqrt{16-\pi^{2}}\right) / 8$, let

$$
h_{*}(x)=f_{*}\left(x+x_{*}\right) \text { if } 0 \leq x \leq 1-x_{*}, \quad h_{*}(x)=f_{*}\left(x+x_{*}-1\right) \text { if } 1-x_{*}<x \leq 1,
$$

and let $\mathcal{H}_{*}(x)$ be the curve defined by $h_{*}$ and the segment $x=x_{*}, 0 \leq y \leq 1-\pi / 8$. Then $\mathcal{H}_{*}(x)$ satisfies the conclusion of the theorem.

The curve $\mathcal{F}_{*}(x)$ and corresponding "generalized thickness" $\mathcal{H}_{*}(x)$ are shown in Figure 1. As is easily seen, $h_{*}$ is obtained as a rearrangement of $f_{*}$ by first taking the part of $f_{*}$ lying above $y=1$ and then appending the part lying below $y=1$.

The theorem shows that to minimize $\mathcal{I}$, it is advantageous to cancel the bulk elastic energy term, which is achieved by breaking the specimen. However, the length of the crack has to be accounted for in the remaining surface energy term. 


\section{Approximation of the Generalized THICKNESS}

By constructing a minimizing sequence, we now show that the value $2+\pi / 4$, given in the previous section as a lower bound for $\inf _{h \in H} \mathcal{I}(h)$, is in fact the value of this quantity.

For $0<\epsilon<1-\pi / 8, \delta>0, \rho>0$, we consider the function $f_{\epsilon, \delta}$ which is continuous on [0,1], linear on $[0, \delta] \cup[1-\delta, 1]$, with value $\epsilon$ at $x=0,1$ and slope $s_{\epsilon, \delta}= \pm(1-\pi / 8+\rho-\epsilon+\sqrt{\delta(1-\delta)}) / \delta$, and for $x \in[\delta, 1-\delta]$,

$$
f_{\epsilon, \delta}(x)=1-\pi / 8+\rho+\sqrt{x(1-x)} .
$$

The constant $\rho$ is selected so that $f_{\epsilon, \delta}$ satisfies the volume constraint

$$
\begin{aligned}
\int_{0}^{1} f_{\epsilon, \delta}= & 2 \int_{0}^{\delta}\left[s_{\epsilon, \delta} x+\epsilon\right] \mathrm{d} x+\int_{\delta}^{1-\delta}[1-\pi / 8+\rho+\sqrt{x(1-x)}] \mathrm{d} x \\
= & \delta[1-\pi / 8+\rho-\epsilon+\sqrt{\delta(1-\delta)}]+(1-2 \delta)(1-\pi / 8+\rho) \\
& +\sqrt{\delta(1-\delta)}(1-2 \delta) / 2+\arcsin (1-2 \delta) / 4+2 \delta \epsilon .
\end{aligned}
$$

The volume constraint yields

$$
\rho=\frac{\delta(1-\epsilon)+\pi / 8(1-\delta)-\sqrt{\delta(1-\delta)} / 2-\arcsin (1-2 \delta) / 4}{1-\delta} .
$$

Expanding $\rho$ as a series in $\delta$ yields $\rho=(1-\pi / 8-\epsilon) \delta+O\left(\delta^{3 / 2}\right)$, so that $\rho$ is positive and tends to 0 as $\delta \rightarrow 0$. Thus, when $\delta$ is small enough, $f_{\epsilon, \delta}$ is an admissible function.

Let us now compute the energy $\mathcal{I}\left(f_{\epsilon, \delta}\right)$. For the surface energy, we have

$$
\int_{0}^{1} \sqrt{1+\left(f_{\epsilon, \delta}^{\prime}\right)^{2}} \mathrm{~d} x=K_{1}+K_{2}
$$

where $K_{1}$ is the length of the linear part, i.e.,

$$
K_{1}=2 \int_{0}^{\delta} \sqrt{1+s_{\epsilon, \delta}^{2}} \mathrm{~d} x=2 \sqrt{\delta^{2}+[1-\pi / 8+\rho-\epsilon+\sqrt{\delta(1-\delta)}]^{2}}
$$

and $K_{2}$ is the length of the arc of the circle, i.e.,

$$
K_{2}=\int_{\delta}^{1-\delta} \sqrt{1+\left(f_{\epsilon, \delta}^{\prime}\right)^{2}} \mathrm{~d} x=\pi / 2-\arccos (1-2 \delta) .
$$

For the elastic part, let

$$
\int_{0}^{1} \frac{1}{f_{\epsilon, \delta}}=J_{1}+J_{2}
$$

where $J_{1}$ corresponds to the linear part, i.e.,

$$
\begin{aligned}
J_{1} & =2 \int_{0}^{\delta} \frac{1}{s_{\epsilon, \delta} x+\epsilon} \mathrm{d} x \\
& =\frac{2 \delta}{1-\pi / 8+\rho-\epsilon+\sqrt{\delta(1-\delta)}} \log \left(1+\frac{1-\pi / 8+\rho-\epsilon+\sqrt{\delta(1-\delta)}}{\epsilon}\right) .
\end{aligned}
$$



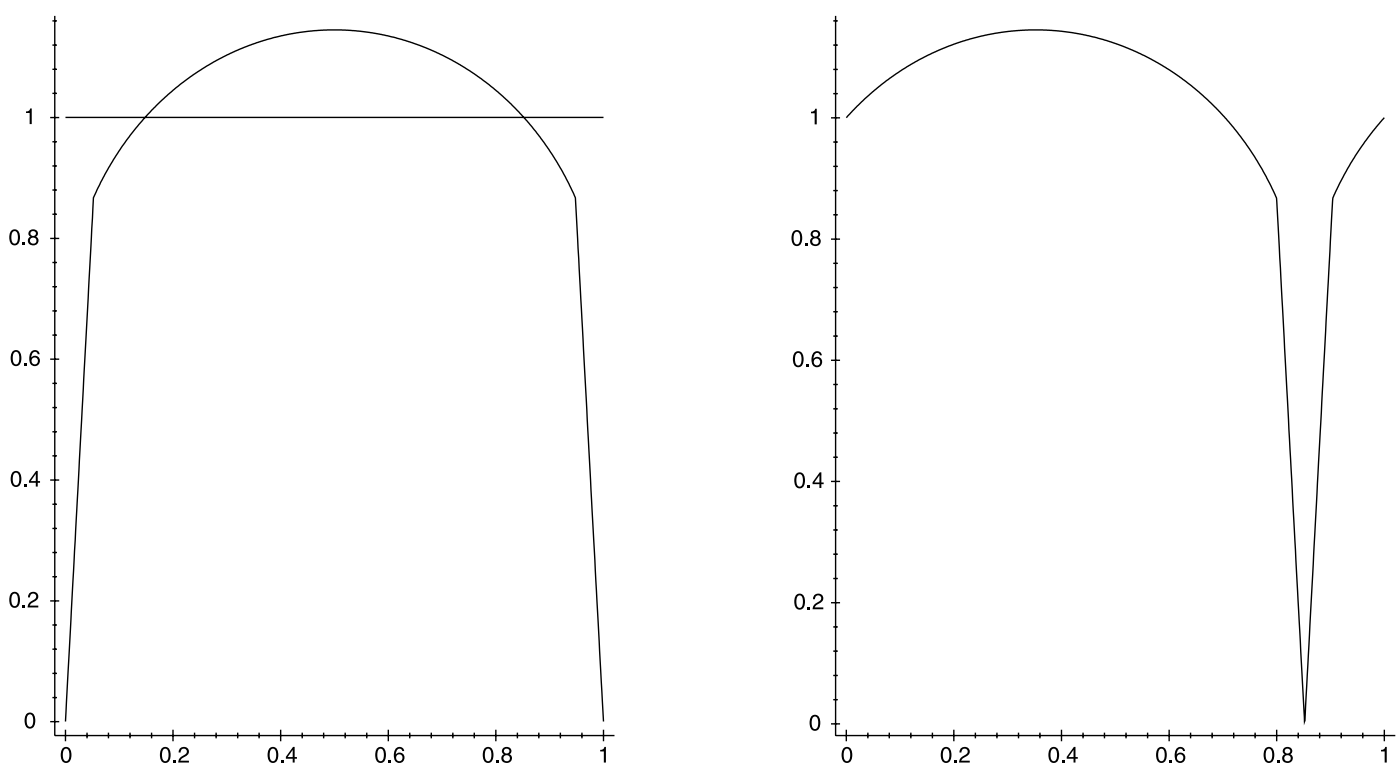

FiguRE 2. Plot of $y=f_{\epsilon, \delta}(x)$ along with $y=1$ and corresponding plot of $y=h_{\epsilon, \delta}(x)$.

The term $J_{2}$ is the contribution of the arc of the circle

$$
0 \leq J_{2}=\int_{\delta}^{1-\delta} \frac{1}{f_{\epsilon, \delta}} \mathrm{d} x \leq \frac{1}{1-\pi / 8}
$$

Thus, the total energy is

$$
\begin{aligned}
\mathcal{I}\left(f_{\epsilon, \delta}\right)= & \pi / 2-\arccos (1-2 \delta)+2 \sqrt{\delta^{2}+[1-\pi / 8+\rho-\epsilon+\sqrt{\delta(1-\delta)}]^{2}} \\
& +N\left[J_{2}+\frac{2 \delta}{1-\pi / 8+\rho-\epsilon+\sqrt{\delta(1-\delta)}} \log \left(1+\frac{1-\pi / 8+\rho-\epsilon+\sqrt{\delta(1-\delta)}}{\epsilon}\right)\right]^{-1}
\end{aligned}
$$

where $J_{2}$ is bounded. When $\rho, \epsilon$, and $\delta$ tend to 0 , this quantity behaves like

$$
\mathcal{I}\left(f_{\epsilon, \delta}\right) \sim \pi / 2+2(1-\pi / 8)+N\left[J_{2}+\frac{2 \delta}{1-\pi / 8} \log \left(\frac{1-\pi / 8}{\epsilon}\right)\right]^{-1}
$$

The choice $\delta=[\log (1 / \epsilon)]^{-1 / 2}$ shows that $I\left(f_{\epsilon, \delta}\right) \rightarrow 2+\pi / 4=I_{2}(0)$, the length of $\mathcal{F}_{*}(x)$, when $\epsilon \rightarrow 0$. On the other hand, the sequence $f_{\epsilon, \delta}$ converges pointwise to $f_{*}$. Therefore, it follows from Theorem 4.1 that $f_{\epsilon, \delta}$ is a minimizing sequence, when $N \geq 2$. 
From $\left(f_{\epsilon, \delta}\right)$, it is then straightforward to construct a minimizing sequence $h_{\epsilon, \delta}$ that satisfies the boundary conditions. Let $h_{\epsilon, \delta}$ be defined by

$$
\begin{aligned}
& h_{\epsilon, \delta}(x)=f_{\epsilon, \delta}\left(x+x_{*}\right) \quad \text { if } 0 \leq x \leq 1-x_{*}, \\
& h_{\epsilon, \delta}(x)=f_{\epsilon, \delta}\left(x+x_{*}-1\right) \quad \text { if } 1-x_{*} \leq x \leq 1 .
\end{aligned}
$$

The function $\left(f_{\epsilon, \delta}\right)$ and corresponding function $h_{\epsilon, \delta}$ are shown in Figure 2 for the choice $\epsilon=10^{-160}$ and $\delta=\log (1 / \epsilon)^{-1 / 2} \approx 0.05$.

For $N \geq 2$, the curve $\mathcal{H}_{*}$ (i.e., $h_{*}$ defined by (4.5) and a crack) is thus a "generalized minimizer" for our original problem.

\section{A RELAXED FORM OF THE ENERGY}

In the result of stability for values of $N \geq 2$, we constructed a sequence of piecewise $C^{1}$ functions $h_{n}$, the energies of which converge to the value of the infimum $2+\pi / 4$. This number is also the length of the non-parametric curve $\mathcal{H}_{*}$, defined in Theorem 4.1. The sequence $\left\{h_{n}\right\}$, satisfies the estimates

$$
\left\|h_{n}\right\|_{L^{1}}=1, \quad \int_{0}^{1}\left|h_{n}^{\prime}\right| \mathrm{d} x \leq \int_{0}^{1} \sqrt{1+\left(h_{n}^{\prime}\right)^{2}} \mathrm{~d} x \leq M
$$

for some constant $M$. In other words, $\left\{h_{n}\right\}$ is a sequence in the space $B V$ of functions of bounded variation [6], which is bounded in the norm in $B V$. It follows that $\left\{h_{n}\right\}$ is precompact in $B V$ [6], i.e., that upon extracting a subsequence, $\left\{h_{n}\right\}$ converges to the $B V$ function $h_{*}$ defined by (4.5):

$$
\begin{aligned}
& h_{*}(x)=1-\pi / 8+\sqrt{\left(x+x_{*}\right)\left(1-x-x_{*}\right)} \quad \text { for } \quad 0 \leq x \leq 1-x_{*}=\left(4+\sqrt{16-\pi^{2}}\right) / 8, \\
& h_{*}(x)=1-\pi / 8+\sqrt{\left(x+x_{*}-1\right)\left(2-x-x_{*}\right)} \quad \text { for } 1-x_{*}<x \leq 1 .
\end{aligned}
$$

The convergence holds in the following sense:

$$
\begin{aligned}
& h_{n} \longrightarrow h_{*} \quad \text { strongly in } L^{1}(0,1), \\
& \liminf _{n \rightarrow \infty} \int_{0}^{1} \sqrt{1+\left(h_{n}^{\prime}\right)^{2}} \mathrm{~d} x \geq \int_{0}^{1} \sqrt{1+\left(h_{*}^{\prime}\right)^{2}} \mathrm{~d} x .
\end{aligned}
$$

We would like to cast the problem of minimizing (1.3) in a setting that ensures well-posedness. In other words, we would like to consider a functional, which is lower semi-continuous in the natural norm, and which is defined on a compact set of admissible thicknesses.

The space $B V$ seems to be the natural space and for $h \in B V$, strictly positive, the definition of $\mathcal{I}(h)$ in $(1.3)$ makes sense. The closure of this subset of $B V$ functions however, contains functions that vanish, for which we need to extend the definition of $\mathcal{I}$. Clearly, the trouble comes from the term $\left(\int_{0}^{1}[h(x)]^{-1} \mathrm{~d} x\right)^{-1}$ that reflects the fact that no uniform coercive estimates on the displacements are available in the original minimization problem (1.1).

Let $H_{*}$ denote the set of positive $B V$ functions, satisfying the boundary conditions and the volume constraint of (1.2). In $H_{*}$, we define

$$
\begin{array}{ll}
\mathcal{J}(h)=\min \left(2 \min (h), \frac{N}{\int_{0}^{1}[h(x)]^{-1} \mathrm{~d} x}\right)+\int_{0}^{1} \sqrt{1+\left(h^{\prime}\right)^{2}} \mathrm{~d} x, & \text { if } \min (h)>0, \\
\mathcal{J}(h)=\int_{0}^{1} \sqrt{1+\left(h^{\prime}\right)^{2}} \mathrm{~d} x, & \text { otherwise. }
\end{array}
$$


Proposition 6.1. The functional $\mathcal{J}$ extends the functional $\mathcal{I}$ in the following sense:

(i) If $h \in H_{*}$ is bounded away from 0 , i.e., $h(x) \geq \alpha>0$ a.e. in $[0,1]$, then $\mathcal{I}(h) \geq \mathcal{J}(h)$.

(ii) If $\left\{h_{n}\right\}$ is a sequence of functions in $H_{*}$ that converges to $h \in H_{*}$ in $L^{1}(0,1)$, such that each $h_{n}$ is bounded away from 0 , then $\liminf _{n \rightarrow \infty} \mathcal{I}\left(h_{n}\right) \geq \mathcal{J}(h)$.

Proof. The first statement is a trivial consequence of the definition of $\mathcal{J}$. To prove the second point, we consider a sequence $\left\{h_{n}\right\} \subset H_{*}$, such that for each $n, \min \left(h_{n}\right)=m_{n}>0$, and $h_{n}(x) \rightarrow h$ in $L^{1}(0,1)$. By density, we can always assume that the functions $h_{n}$ are $C^{1}$ on $[0,1][6]$.

Case 1. If $\min (h)=0$, then

$$
\liminf _{n \rightarrow \infty} \mathcal{I}\left(h_{n}\right) \geq \liminf _{n \rightarrow \infty} \int_{0}^{1} \sqrt{1+\left(h_{n}^{\prime}\right)^{2}} \mathrm{~d} x \geq \int_{0}^{1} \sqrt{1+\left(h^{\prime}\right)^{2}} \mathrm{~d} x=\mathcal{J}(h),
$$

where the last inequality follows from the lower semi-continuity of $\int_{0}^{1} \sqrt{1+\left(h^{\prime}\right)^{2}} \mathrm{~d} x$ (i.e., the length of $h$ ) in $B V[6]$.

Case 2. If $\min (h)=m>0$ and $\liminf _{n \rightarrow \infty} m_{n}>0$, then, $h^{-1} \in L^{1}(0,1)$ and for a subsequence

$$
h_{n} \rightarrow h \text { a.e., } \quad h_{n}^{-1} \rightarrow h^{-1} \text { a.e. }
$$

From the Lebesgue Dominated Convergence Theorem, it follows that

$$
\frac{N}{\int_{0}^{1} h_{n}^{-1} \mathrm{~d} x} \rightarrow \frac{N}{\int_{0}^{1} h^{-1} \mathrm{~d} x} .
$$

Thus, using again the lower semi-continuity of the length in $B V$, we obtain

$$
\liminf _{n \rightarrow \infty} \mathcal{I}\left(h_{n}\right) \geq \frac{N}{\int_{0}^{1} h^{-1} \mathrm{~d} x}+\int_{0}^{1} \sqrt{1+\left(h^{\prime}\right)^{2}} \mathrm{~d} x \geq \mathcal{J}(h) .
$$

Case 3. If $\min (h)=m>0$ and $\liminf _{n \rightarrow \infty} m_{n}=0$, then we can always assume that the whole sequence $h_{n}$ tends to $h$ a.e. and that

$$
m_{n} \rightarrow 0
$$

Let $\epsilon>0$ be such that $m-\epsilon>m / 2>0$. For $n$ larger than some $N_{0}, m_{n}+\epsilon<m-\epsilon$. Let $\phi_{n}(x)=$ $\sup \left(m-\epsilon, h_{n}(x)\right)$. Since $h_{n} \leq \phi_{n} \leq \sup \left(h, h_{n}\right)$ a.e.,

$$
\phi_{n} \rightarrow h \quad \text { a.e. }
$$

Moreover, since $h_{n}$ is continuous, for $n>N_{0}$ there exists an interval $\left[x_{n}, y_{n}\right]$, of length $d_{n}$, such that

$$
h_{n}\left(x_{n}\right)=h_{n}\left(y_{n}\right)=m-\epsilon, \quad \min \left(h_{n}\right)=m_{n} \text { in }\left[x_{n}, y_{n}\right], \quad h_{n} \leq m-\epsilon \text { in }\left[x_{n}, y_{n}\right] .
$$

The length of $h_{n}$ on $\left[x_{n}, y_{n}\right]$ is greater than the length of two straight lines connecting the points $\left(x_{n}, m-\right.$ $\epsilon),\left(\left[x_{n}+y_{n}\right] / 2, m_{n}\right),\left(y_{n}, m-\epsilon\right)$. Hence,

$$
\int_{x_{n}}^{y_{n}} \sqrt{1+\left(h_{n}^{\prime}\right)^{2}} \mathrm{~d} x \geq \sqrt{d_{n}^{2}+4\left(m-\epsilon-m_{n}\right)^{2}}
$$


On the other hand, since the length of $\phi_{n}$ on that segment is simply $d_{n}$, we get

$$
\int_{0}^{1} \sqrt{1+\left(h_{n}^{\prime}\right)^{2}} \mathrm{~d} x \geq \int_{0}^{1} \sqrt{1+\left(\phi_{n}^{\prime}\right)^{2}} \mathrm{~d} x+\sqrt{d_{n}^{2}+4\left(m-\epsilon-m_{n}\right)^{2}}-d_{n} .
$$

We claim that

$$
d_{n} \rightarrow 0 \text { as } n \rightarrow \infty
$$

Indeed, if $d_{n} \rightarrow \alpha>0$, we could find a subsequence such that $x_{n} \rightarrow x, y_{n} \rightarrow y$, and for $n$ large enough,

$$
x_{n}-\alpha / 5<x<x_{n}+\alpha / 5<y_{n}-\alpha / 5<y<y_{n}+\alpha / 5
$$

so that we would have $h_{n} \leq m-\epsilon$ on $[x+\alpha / 5, y-\alpha / 5]$ for $n$ large enough. This contradicts the fact that $h_{n} \rightarrow h$ a.e.

Finally, using (6.1-6.2) and the semi-continuity of the length in $B V$, we have

$$
\begin{aligned}
\liminf _{n \rightarrow \infty} \mathcal{I}\left(h_{n}\right) & \geq \liminf _{n \rightarrow \infty} \int_{0}^{1} \sqrt{1+\left(h_{n}^{\prime}\right)^{2}} \mathrm{~d} x \\
& \geq \liminf _{n \rightarrow \infty}\left[\int_{0}^{1} \sqrt{1+\left(\phi_{n}^{\prime}\right)^{2}} \mathrm{~d} x+\sqrt{d_{n}^{2}+4\left(m-\epsilon-m_{n}\right)^{2}}-d_{n}\right] \\
& \geq \int_{0}^{1} \sqrt{1+\left(h^{\prime}\right)^{2}} \mathrm{~d} x+2(m-\epsilon) .
\end{aligned}
$$

Letting $\epsilon$ tend to 0 , we obtain $\liminf _{n \rightarrow \infty} \mathcal{I}\left(h_{n}\right) \geq \mathcal{J}(h)$.

Proposition 6.2. The functional $\mathcal{J}$ is lower semi-continuous on $H_{*}$.

Proof. Let $\left\{h_{n}\right\} \subset H_{*}$ be such that $h_{n} \rightarrow h$ in $L^{1}(0,1)$ and $\int \sqrt{1+\left(h_{n}^{\prime}\right)^{2}} \mathrm{~d} x$ is bounded. We want to show that

$$
\liminf _{n \rightarrow \infty} \mathcal{J}\left(h_{n}\right) \geq \mathcal{J}(h)
$$

We can always assume that the functions $h_{n}$ are $C^{1}[6]$.

If $\min (h)=0$, then $(6.3)$ is satisfied trivially. If $\min (h)=m>0$ and $\min \left(h_{n}\right)=m_{n}$ tends to some value $m^{*} \geq m$, then

$$
\min \left(2 m_{n}, \frac{N}{\int_{0}^{1}\left[h_{n}\right]^{-1} \mathrm{~d} x}\right) \rightarrow \min \left(2 m^{*}, \frac{N}{\int_{0}^{1} h^{-1} \mathrm{~d} x}\right) \geq \min \left(2 m, \frac{N}{\int_{0}^{1} h^{-1} \mathrm{~d} x}\right)
$$

and (6.3) follows from the lower semi-continuity of the length.

If $m^{*}<m$, then let $\epsilon>0$ be such that $m^{*}+\epsilon<m-\epsilon$ and let $\phi_{n}(x)=\sup \left(m-\epsilon, h_{n}(x)\right)$. Then, by the same arguments as those of Proposition 6.1,

$$
\begin{aligned}
\liminf _{n \rightarrow \infty} \int_{0}^{1} \sqrt{1+\left(h_{n}^{\prime}\right)^{2}} \mathrm{~d} x & \geq \liminf _{n \rightarrow \infty}\left[\int_{0}^{1} \sqrt{1+\left(\phi_{n}^{\prime}\right)^{2}} \mathrm{~d} x+\sqrt{d_{n}^{2}+4\left(m-\epsilon-m_{n}\right)^{2}}-d_{n}\right] \\
& \geq \int_{0}^{1} \sqrt{1+\left(h^{\prime}\right)^{2}} \mathrm{~d} x+2\left(m-\epsilon-m^{*}\right) .
\end{aligned}
$$

If $m^{*}=0$, since $\liminf _{n \rightarrow \infty} \mathcal{J}\left(h_{n}\right)$ is larger than the right-hand side of the above inequality, (6.3) is obtained by letting $\epsilon \rightarrow 0$. 
If $m^{*}>0$, then we get

$$
\liminf _{n \rightarrow \infty}\left[2 m_{n}+\int_{0}^{1} \sqrt{1+\left(h_{n}^{\prime}\right)^{2}} \mathrm{~d} x\right] \geq \mathcal{J}(h)-2 \epsilon .
$$

On the other hand, the Dominated Convergence Theorem and the lower semi-continuity of the length yield

$$
\liminf _{n \rightarrow \infty}\left[\frac{N}{\int_{0}^{1}\left[h_{n}\right]^{-1} \mathrm{~d} x}+\int_{0}^{1} \sqrt{1+\left(h_{n}^{\prime}\right)^{2}} \mathrm{~d} x\right] \geq \frac{N}{\int_{0}^{1} h^{-1} \mathrm{~d} x}+\int_{0}^{1} \sqrt{1+\left(h^{\prime}\right)^{2}} \mathrm{~d} x \geq \mathcal{J}(h),
$$

and (6.3) follows from these last two inequalities by letting $\epsilon$ tend to 0 .

Proposition 6.2 and the precompactness of sequences in $H_{*}$ in the norm in $B V$ imply that $\mathcal{J}$ achieves a minimum in $H_{*}$. For $N \geq 2$,

$$
\frac{N}{\int_{0}^{1} h^{-1} \mathrm{~d} x} \geq \frac{N}{\int_{0}^{1}[\min (h)]^{-1} \mathrm{~d} x} \geq 2 \min (h)
$$

and thus,

$$
\forall h \in H_{*}, \quad \mathcal{J}(h)=2 \min (h)+\int_{0}^{1} \sqrt{1+\left(h^{\prime}\right)^{2}} \mathrm{~d} x .
$$

The arguments of Section 4 show not only that $\mathcal{I}(h) \geq 2+\pi / 4$, but also that $\mathcal{J}(h) \geq 2+\pi / 4$. On the other hand, the function $h_{*}$, defined by (4.5) satisfies

$$
\mathcal{J}\left(h_{*}\right)=2+\pi / 4=\min \mathcal{J} .
$$

\section{Appendix}

Proof of Proposition 4.2. Assume $f \in F_{e_{0}}$, i.e., that $f$ is a piecewise $C^{1}$ function such that

$$
f(0)=f(1)=e_{0}, \quad f(x) \geq e_{0}, \quad x \in[0,1], \quad \int_{0}^{1} f(x) \mathrm{d} x=1 .
$$

Since the minimal length of $f \in F_{e_{0}}$ depends on the value of $e_{0}$, we shall consider two cases.

Case 1. $1-\pi / 8 \leq e_{0} \leq 1$.

In this case, we first show that there is an arc of a circle which is an admissible curve. To this effect, we seek $y_{0}$ and $R_{e_{0}}$, such that

$$
f_{0}(x)=y_{0}+\sqrt{R_{e_{0}}^{2}-(x-1 / 2)^{2}}
$$

defines an arc of a circle that connects the point $\left(0, e_{0}\right)$ to $\left(1, e_{0}\right)$, that encloses an area equal to 1 , and that lies above the level $e_{0}$. Expressing these conditions, we get

$$
\begin{aligned}
\left(e_{0}-y_{0}\right) & =\sqrt{R_{e_{0}}^{2}-1 / 4}, \\
1=\int_{0}^{1}\left[y_{0}+\sqrt{R_{e_{0}}^{2}-(x-1 / 2)^{2}}\right] \mathrm{d} x & =y_{0}+\frac{\sqrt{4 R_{e_{0}}^{2}-1}}{4}+R_{e_{0}}^{2} \arcsin \left(1 /\left[2 R_{e_{0}}\right]\right) .
\end{aligned}
$$


It follows that

$$
e_{0}=1+(1 / 4) \sqrt{4 R_{e_{0}}^{2}-1}-R_{e_{0}}^{2} \arcsin \left(1 /\left[2 R_{e_{0}}\right]\right)
$$

For $r \in(1 / 2, \infty)$, let

$$
\phi(r)=1+(1 / 4) \sqrt{4 r^{2}-1}-r^{2} \arcsin (1 /[2 r]) .
$$

Then $\phi^{\prime}(r)=2 r \rho(r)$ where $\rho(r)=\left(4 r^{2}-1\right)^{-1 / 2}-\arcsin (1 /[2 r])$. Since $\rho^{\prime}(r)=-1 /\left[r\left(4 r^{2}-1\right)^{3 / 2}\right]<0$ for $r>1 / 2, \rho$ is a decreasing function. Since $\rho$ tends to zero as $r$ tends to infinity, it follows that $\rho$ and hence $\phi^{\prime}(r)$ are positive, which implies that $\phi$ is a strictly increasing function. It is easy to check that $\phi$ maps $(1 / 2, \infty)$ onto $(1-\pi / 8,1)$. Thus, for each $1-\pi / 8<e_{0}<1$, there is a unique $R_{e_{0}}$ solution of (7.1). Furthermore, $R_{e_{0}}$ tends to $1 / 2$ (resp. $\infty)$ as $e_{0}$ tends to $1-\pi / 8$ (resp. 1 ).

Let $D_{0}$ denote the upper half of the disc of radius $R_{e_{0}}$, centered at $\left(1 / 2, y_{0}\right)$, and let $\Gamma_{0}$ denote the part of its boundary that lies below $e_{0}$ and above $y_{0}$. The domain $D$ enclosed by $\Gamma_{0}$, the line $y=y_{0}$, and the curve defined by $f$ has the same area as $D_{0}$. The classical isoperimetric inequality [2] implies that the length of the boundary of $D$ is greater or equal to the length of the boundary of $D_{0}$. Thus

$$
\int_{0}^{1} \sqrt{1+\left(f^{\prime}\right)^{2}} \mathrm{~d} x \geq \int_{0}^{1} \sqrt{1+\left(f_{0}^{\prime}\right)^{2}} \mathrm{~d} x=2 R_{e_{0}} \arcsin \left(1 /\left[2 R_{e_{0}}\right]\right) .
$$

Case 2. $0 \leq e_{0}<1-\pi / 8$.

We can no longer draw an arc of a circle bounding an area of 1 through the points $\left(0, e_{0}\right)$ and $\left(1, e_{0}\right)$. The proof of this case is divided into two steps. In the first one, we replace $f$ by another function $f_{*}$ that has length less than or equal to the length of $f$. Then in the second step, we obtain a lower bound on the length of $f_{*}$.

Step 1:

In addition to the previous hypotheses, assume that $f$ is piecewise linear. We shall subsequently extend the results obtained by a density argument. Let $e_{M}$ be the maximum of $f$. For $e \in\left[e_{0}, e_{M}\right]$, we define

$$
\Omega_{e}=\{0 \leq x \leq 1: f(x) \geq e\}, \quad g(e)=\int_{\Omega_{e}}(f-e) \mathrm{d} x, \quad h(e)=\pi / 8\left|\Omega_{e}\right|^{2} .
$$

The function $h$ is the area of a half circle of diameter $\left|\Omega_{e}\right|$. It is a right-continuous, decreasing function. The function $g$ measures the area enclosed by $f$ above the level $e$. One can readily check that $g$ is decreasing and continuous: if $e<e^{\prime}$,

$$
\begin{aligned}
g(e) & =\int_{\Omega_{e}^{\prime}}(f-e) \mathrm{d} x+\int_{\Omega_{e} \backslash \Omega_{e}^{\prime}}(f-e) \mathrm{d} x \\
& \leq \int_{\Omega_{e}^{\prime}}\left(f-e^{\prime}\right) \mathrm{d} x+\int_{\Omega_{e}^{\prime}}\left(e^{\prime}-e\right) \mathrm{d} x+\left(e^{\prime}-e\right)\left|\Omega_{e} \backslash \Omega_{e}^{\prime}\right| \\
& \leq g\left(e^{\prime}\right)+\left(e^{\prime}-e\right) .
\end{aligned}
$$

Also, we have

$$
g\left(e_{0}\right)=1-e_{0}>\pi / 8=h\left(e_{0}\right)
$$

Again, we consider several cases.

Case $a: h(e) \geq g(e)$ for some value of $e \in\left(e_{0}, e_{M}\right)$ or $h\left(e_{M}\right)>g\left(e_{M}\right)$.

The motonicity and continuity properties of $g$ and $h$, together with (7.2), imply that $h\left(e_{*}\right)=g\left(e_{*}\right)$, for some value $e_{*} \in\left(e_{0}, e_{M}\right)$. 
Since the area enclosed by $f$ and the length of $f$ are translation invariant, $\Omega_{e_{*}}$ can be assumed to be connected and centered at some point $x_{*}$. Then, the function $f_{*}$, given by

$$
\begin{array}{ll}
f_{*}(x)=f(x) & \text { if } x \in[0,1] \backslash \Omega_{e_{*}}, \\
f_{*}(x)=e_{*}+\sqrt{\left|\Omega_{e_{*}}^{2}\right| / 4-\left(x-x_{*}\right)^{2}} & \text { if } x \in \Omega_{e_{*}}
\end{array}
$$

also encloses an area equal to 1 . It is a consequence of the standard isoperimetric inequality on $\Omega_{e_{*}}$, that $f_{*}$ has a smaller length than $f$.

Case b: $h(e)<g(e)$ for all $e \in\left(e_{0}, e_{M}\right)$ and $h\left(e_{M}\right) \leq g\left(e_{M}\right)$.

First, observe that if $\Omega_{e_{M}}$ contains a subset $\omega$ where $f$ is flat, then $g\left(e_{M}\right)=0$, while $h\left(e_{M}\right) \geq \pi / 8|\omega|^{2}>0$. Since this cannot occur under the hypothesis of Case $\mathrm{b}$, we conclude that $f^{\prime} \neq 0$ a.e. in $\Omega_{e_{M}}$. Hence, for $e$ close enough to $e_{M}, \Omega_{e}$ consists of a finite number of intervals

$$
\Omega_{e}=\cup_{1 \leq i \leq N}\left[x_{i}-r_{i}^{-}, x_{i}+r_{i}^{+}\right],
$$

such that $f$ is increasing on $\left[x_{i}-r_{i}^{-}, x_{i}\right]$ from $f(e)$ to $f\left(e_{M}\right)$ and decreasing on $\left[x_{i}, x_{i}+r_{i}^{+}\right]$from $f\left(e_{M}\right)$ to $f(e)$. Again, by translation invariance, $\Omega_{e}$ can be assumed to be connected (i.e., $\left.x_{i}+r_{i}^{+}=x_{i+1}-r_{i+1}^{-}\right)$and centered at some point $x_{*}$. Since $f$ is piecewise linear and has a saw-tooth profile in $\Omega_{e}$, we have

$$
\int_{\Omega_{e}}(f-e) \mathrm{d} x=\left|\Omega_{e}\right|\left(e_{M}-e\right) / 2
$$

Thus, $h(e)<g(e)$ implies that $\left(e_{M}-e\right) / 2-\pi / 8\left|\Omega_{e}\right|$ is positive. Hence,

$$
e_{*} \equiv e+\left(e_{M}-e\right) / 2-\pi / 8\left|\Omega_{e}\right|=\left(e_{M}+e\right) / 2-\pi / 8\left|\Omega_{e}\right|>e .
$$

Clearly, we also have $e_{*}<e_{M}$. Let $f_{*}(x)$ be the function defined by

$$
\begin{aligned}
& f_{*}(x)=f(x) \quad \text { if } x \in[0,1] \backslash \Omega_{e}, \\
& f_{*}(x)=e_{*}+\sqrt{\left|\Omega_{e}\right|^{2} / 4-\left(x-x_{*}\right)^{2}} \quad \text { if } x \in \Omega_{e}, \text { i.e., }\left|x-x_{*}\right| \leq\left|\Omega_{e}\right| / 2,
\end{aligned}
$$

and let $C_{*}$ be the curve defined by the union of the half circle $\left(x, f_{*}(x)\right),\left|x-x_{*}\right| \leq\left|\Omega_{e}\right| / 2$, and the two vertical segments $\left[x_{*} \pm\left|\Omega_{e}\right| / 2, y\right], e \leq y \leq e_{*}$.

According to the definition of $e_{*}$,

$$
\begin{aligned}
\int_{\Omega_{e}}\left(f_{*}-e\right) \mathrm{d} x & =\int_{\Omega_{e}}\left[\left(e_{*}-e\right)+\sqrt{\left|\Omega_{e}\right|^{2} / 4-\left(x-x_{*}\right)^{2}}\right] \mathrm{d} x=\left(e_{*}-e\right)\left|\Omega_{e}\right|+\pi\left|\Omega_{e}\right|^{2} / 8 \\
& =\left[\left(e_{M}-e\right) / 2-\pi\left|\Omega_{e}\right| / 8\right]\left|\Omega_{e}\right|+\pi\left|\Omega_{e}\right|^{2} / 8=\left|\Omega_{e}\right|\left(e_{M}-e\right) / 2=\int_{\Omega_{e}}(f-e) \mathrm{d} x .
\end{aligned}
$$

Besides, (7.3) gives the following estimate of $\left|\Omega_{e}\right|$.

$$
h(e)=\pi\left|\Omega_{e}\right|^{2} / 8<g(e)=\left|\Omega_{e}\right|\left(e_{M}-e\right) / 2 \quad \text { i.e., } \quad \pi\left|\Omega_{e}\right| / 4<e_{M}-e .
$$


Now the length of $f$ on $\Omega_{e}$ is given by

$$
\begin{aligned}
\sum_{i=1}^{N}\left[\sqrt{\left(r_{i}^{-}\right)^{2}+\left(e_{M}-e\right)^{2}}\right. & \left.+\sqrt{\left(r_{i}^{+}\right)^{2}+\left(e_{M}-e\right)^{2}}\right] \\
& =\sum_{i=1}^{N}\left(\frac{r_{i}^{-}}{\left|\Omega_{e}\right|} \sqrt{\left|\Omega_{e}\right|^{2}+\left[\frac{\left|\Omega_{e}\right|}{r_{i}^{-}}\left(e_{M}-e\right)\right]^{2}}+\frac{r_{i}^{+}}{\left|\Omega_{e}\right|} \sqrt{\left|\Omega_{e}\right|^{2}+\left[\frac{\left|\Omega_{e}\right|}{r_{i}^{+}}\left(e_{M}-e\right)\right]^{2}}\right) \\
& \geq \sqrt{\left|\Omega_{e}\right|^{2}+4 N^{2}\left(e_{M}-e\right)^{2}} \geq \sqrt{\left|\Omega_{e}\right|^{2}+4\left(e_{M}-e\right)^{2}}
\end{aligned}
$$

by the convexity of the function $\sqrt{a^{2}+x^{2}}$. On the other hand, using (7.4), the length of $C_{*}$ is

$$
2\left(e_{*}-e\right)+\pi\left|\Omega_{e}\right| / 2=\left(e_{M}-e\right)+\pi\left|\Omega_{e}\right| / 4 \leq 2\left(e_{M}-e\right)<\sqrt{\left|\Omega_{e}\right|^{2}+4\left(e_{M}-e\right)^{2}},
$$

and thus is smaller then the length of $f$.

Step 2:

So far, given a piecewise linear admissible function $f$, we have constructed another admissible function $f_{*}$, which may have jumps, but whose length, $l\left(f_{*}\right)$, is less than or equal to the length of $f$. In particular, the constraint on the area yields

$$
1=\int_{\left\{f_{*} \geq e_{*}\right\}} f_{*} \mathrm{~d} x+\int_{[0,1] \backslash\left\{f_{*} \geq e_{*}\right\}} f_{*} \mathrm{~d} x .
$$

In Case a, $\Omega_{e_{*}}$ is also the set where $f_{*} \geq e_{*}$ and so it follows easily from the above that

$$
1 \leq \int_{\Omega_{e_{*}}} f_{*} \mathrm{~d} x+e_{*}\left(1-\left|\Omega_{e_{*}}\right|\right)=(\pi / 8)\left|\Omega_{e_{*}}\right|^{2}+e_{*}\left|\Omega_{e_{*}}\right|+e_{*}\left(1-\left|\Omega_{e_{*}}\right|\right) \leq(\pi / 8)+e_{*} .
$$

In Case $\mathrm{b}, \Omega_{e}$ is the set where $f_{*} \geq e_{*}$ and so

$$
1 \leq \int_{\Omega_{e}} f_{*} \mathrm{~d} x+e_{*}\left(1-\left|\Omega_{e}\right|\right)=(\pi / 8)\left|\Omega_{e}\right|^{2}+e_{*}\left|\Omega_{e}\right|+e_{*}\left(1-\left|\Omega_{e}\right|\right) \leq(\pi / 8)+e_{*} .
$$

Hence, in both cases,

$$
e_{*} \geq 1-\pi / 8
$$

Let $D$ be the domain that consists of the area enclosed by $f_{*}$ above the level $e_{0}$ and its symmetric image about the line $y=e_{0}$. Its area is $\mathcal{A}(D)=2\left(1-e_{0}\right)$, and its length is $l(D)=2 l\left(f_{*}\right)$. Further, by construction, $D$ contains two discs of radius $\left|\Omega_{e_{*}}\right| / 2$, whose centers are separated by a distance $d=2\left(e_{*}-e_{0}\right)$. In this situation, the following isoperimetric inequality holds (see p. 7 in [2]).

$$
l(D)^{2} \geq 4 \pi \mathcal{A}(D)+4 d^{2}
$$

i.e.,

$$
l\left(f_{*}\right)^{2} \geq 2 \pi\left(1-e_{0}\right)+4\left(e_{*}-e_{0}\right)^{2} \geq 2 \pi\left(1-e_{0}\right)+4\left(1-\pi / 8-e_{0}\right)^{2}=\left[2\left(1-e_{0}\right)+\pi / 4\right]^{2} .
$$

By density, it follows that for all $f \in F_{e_{0}}$

$$
\int_{0}^{1} \sqrt{1+\left(f^{\prime}\right)^{2}} \mathrm{~d} x \geq 2\left(1-e_{0}\right)+\pi / 4
$$


It is easy to check that this value is attained by the curve consisting of a half circle of radius $1 / 2$ centered at $\left(1 / 2, e_{*}\right)$ and the two vertical segments $[0, y]$, and $[1, y]$, with $e_{0} \leq y \leq e_{*}$, where $e_{*}=1-\pi / 8$. Note that this is precisely the curve $C_{*}$ in the case when $e=e_{0}$ (and $\Omega_{e}=[0,1]$ ).

\section{REFERENCES}

[1] J.M. Ball and V.J. Mizel, One-dimensional variational problems whose minimizers do not satisfy the Euler Lagrange equation. Arch. Rational Mech. Anal. 90 (1985) 325-388.

[2] C. Bandle, Isoperimetric Inequalities and Applications. Pitman (1980).

[3] B. Dacorogna, Direct Methods in the Calculus of Variations. Springer-Verlag, Berlin, New York (1989).

[4] Solids Far From Equilibrium. C. Godreche Ed., Cambridge University Press (1991).

[5] J.W. Gibbs, On the equilibrium of heterogeneous substances. Trans. Connect. Acad. Sci. 3 (1876-1878) 108-248, 343-524.

[6] E. Giusti, Minimal surfaces and functions of bounded variation. Birkhauser, Boston (1984).

[7] M.A. Grinfeld, Thermodynamic Methods in the Theory of Heterogeneous Systems. Longman (1991).

[8] M.A. Grinfeld, Stress driven instabilities in crystals: mathematical models and physical manifestations. J. Nonlinear Sci. 3 (1993) 35-83.

[9] M.A. Grinfeld, Manifestations of the stress driven rearrangement instabilities in creep, damaging and fracture. Final Report. Wright-Patterson Laboratory (1995).

[10] MSR Bulletin (1996).

[11] L.D. Landau and E.M. Lifshitz, Statistical physics, Part 1. Oxford, Pergamon Press (1986).

[12] P. Nozières, Lectures at Collège de France (1988) unpublished notes. Growth and Shape of Crystals (lectures given at Beg-Rohu Summer School, Brittany, 1989) in Solids Far From Equilibrium, C. Godreche Ed., Cambridge University Press (1991).

[13] P. Nozières, Amplitude Expansion for the Grinfeld Instability due to Uniaxial Stress at a Solid Surface. J. Phys. 3 (1993) 681. 